\title{
Discrimination of Isomeric Carbohydrates as the Electron Transfer Products of Group II Cation Adducts by Ion Mobility Spectrometry and Tandem Mass Spectrometry
}

\author{
Yuting Huang and Eric D. Dodds* \\ Department of Chemistry, University of Nebraska-Lincoln, Lincoln, Nebraska 68588-0304, United States
}

ABSTRACT: The rapid and unambiguous distinction of isomeric carbohydrate structures persists as a tremendous analytical challenge. This paper reports the first exploitation of carbohydrate/metal ion interactions in concert with gas-phase ion chemistry to improve discrimination of oligosaccharide isomers by both ion mobility spectrometry and tandem mass spectrometry. This is demonstrated for two isomeric pentasaccharides and two isomeric hexasaccharides, each studied in an underivatized form as their calcium ion adducts, barium ion adducts, and gas-phase electron transfer products thereof. With appropriate selection of the charge carrier,
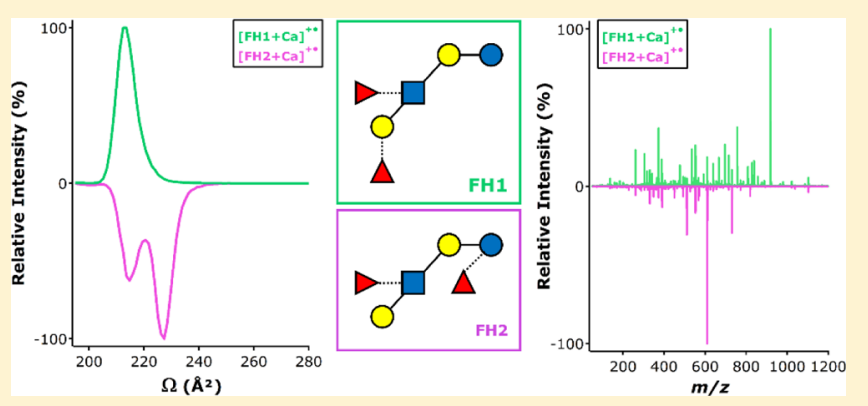
transfer of a single electron to the carbohydrate metal ion adducts resulted in isomer-distinguishing shifts in their ion/neutral collision cross sections and the appearance of unique features in their vibrational activation/dissociation spectra. These findings suggest novel and elegant gas-phase strategies for rapid differentiation of isomeric oligosaccharides.

$\mathrm{T}$ he wide-ranging biochemical significance of oligosaccharides, coupled with the high molecular complexity of their fine structures, have stimulated high demand for comprehensive glycoanalytical tools. ${ }^{1,2}$ Chemical and physical methods capable of rapidly differentiating isomeric carbohydrates are of particular interest. While a wide variety of analytical approaches have been applied to structural determination of oligosaccharides, mass spectrometry (MS) has proven an especially powerful platform for many aspects of carbohydrate analysis. ${ }^{3,4}$ Unfortunately, the ability of MS-based methods to expeditiously and unambiguously distinguish oligosaccharide isomers remains sorely limited. ${ }^{5,6}$ However, MS coupled with ion mobility spectrometry (IMS) and certain ion fragmentation methods for tandem mass spectrometry (MS/MS) has shown potential to play an expanded role in the rapid discrimination of carbohydrate isomers. IMS is attractive in this context because gas-phase ion mobilities largely depend on orientationally averaged ion-neutral collision cross sections (CCSs), which can be sensitive to structural differences among isomers. ${ }^{7}$ This presents significant opportunities for the detection, distinction, and resolution of isomeric saccharides on a millisecond timescale. $^{8}$ Nevertheless, full resolution of isomeric species in mixtures remains challenging, particularly for larger oligosaccharides. ${ }^{9,10}$ In addition, while MS/MS fragmentation methods can contribute to rapid elucidation of the general connectivity of monosaccharides within a glycan structure, isomeric species tend to undergo a shared unimolecular dissociation process to yield fragments with identical mass. ${ }^{112}$ This can seriously limit the ability of MS/MS methods to differentiate isomers.

An opportunity for extending the capabilities of both IMS and MS/MS for carbohydrate structure analysis lies in the propensity of carbohydrates to bind a variety of cations. The metal ion adduction of glycans has been noted to affect the ion mobilities and fragmentation behavior of these species in cation-dependent and structurally relevant manners. ${ }^{13,14}$ Collectively, these observations point to a role for the charge carrier as a probe of carbohydrate structure in conjunction with both IMS and MS/MS. Here, we extend this notion through the use of gas-phase ion chemistry to expand the catalog of such probes and demonstrate their yet untapped potential for carbohydrate isomer discrimination.

The presently described experiments were focused on two pairs of isomeric carbohydrates: the pentasaccharide isomers lacto- $N$-fucopentaose I (FP1) and lacto- $N$-fucopentaose V (FP5); and the hexasaccharide isomers lacto- $N$-difucohexaose I (FH1) and lacto- $\mathrm{N}$-difucohexaose II (FH2). These oligosaccharide isomers were selected for study because they have proven difficult to distinguish by both $\mathrm{IMS}^{15,16}$ and MS/ $\mathrm{MS}^{17,18}$ analysis. The structures of these carbohydrates are schematically diagrammed in Figure 1 with use of the monosaccharide symbols of Varki et al. ${ }^{19}$ and the glycosidic linkage conventions of Harvey et al. ${ }^{20}$ Both pairs of isomers differed from each other only in the connectivity of a single fucose residue: either $\alpha(1-2)$ linked to the nonreducing terminal galactose residue (as in FP1 and FH1) or $\alpha(1-3)$ linked to the reducing terminal glucose residue (as in FP5 and $\mathrm{FH} 2$ ). Separate solutions of each oligosaccharide were prepared at $5 \mu \mathrm{M}$ in $50 \% \mathrm{v} / \mathrm{v}$ aqueous acetonitrile, which also contained

Received: February 25, 2015

Accepted: May 8, 2015

Published: May 8, 2015 

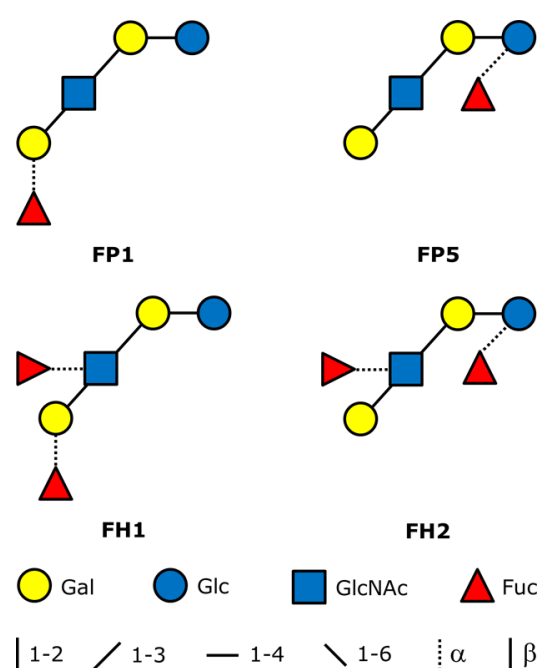

FP5

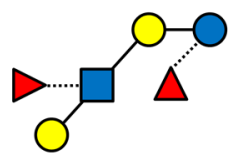

Figure 1. Schematic representations of the pentasaccharide isomers lacto- $N$-fucopentaose I (FP1) and lacto- $N$-fucopentaose V (FP5), and the hexasaccharide isomers lacto- $\mathrm{N}$-difucohexaose I (FH1) and lacto$\mathrm{N}$-difucohexaose II (FH2). A key to monosaccharide symbols and glycosidic linkages is provided.

either $50 \mu \mathrm{M} \mathrm{CaCl}_{2}$ or $50 \mu \mathrm{M} \mathrm{BaCl}_{2}$ in order to produce the corresponding metal ion adduct. These solutions were analyzed using nanoelectrospray ionization (nESI) interfaced to a quadrupole time-of-flight ( $\mathrm{Q}-\mathrm{TOF}$ ) hybrid tandem mass spectrometer capable of traveling wave ion mobility spectrometry (TWIMS) and equipped to carry out gas-phase ion-ion electron transfer (ET) reactions. When desired, ET reactions were carried out using the radical anion of 1,4-dicyanobenzene, produced by glow discharge, as the electron donor. For a carbohydrate $(\mathrm{C})$ cationized by adduction of a metal ion (M, carrying $n$ charges), ET from the reagent (R) to the carbohydrate metal ion adduct proceeded according to

$$
[\mathrm{C}+\mathrm{M}]^{n+}+\mathrm{R}^{-\bullet} \rightarrow[\mathrm{C}+\mathrm{M}]^{(n-1)+\bullet}+\mathrm{R}
$$

These ET reactions were carried out in the pre-mobility stacked ring ion guide (SRIG) of the instrument using helium as the buffer gas. The pressure of helium during ET reactions was maintained at sufficient levels $\left(>5 \cdot 10^{-2} \mathrm{mbar}\right)$ to vibrationally cool the ET products such that they did not undergo electron transfer dissociation (ETD) $)^{21}$ but remained intact over the timescale of IMS measurements $(\sim 10 \mathrm{~ms})$, unless deliberately vibrationally activated. The CCSs of analyte ions were measured by calibrating TWIMS drift times to CCS using protonated polyalanine ions with analyte-matched charge states as standards. ${ }^{22,23}$ MS/MS was conducted using collisioninduced dissociation (CID) of analyte ions at various acceleration potentials $(\Delta U)$ and employing argon as the collision gas. For the doubly charged carbohydrate metal ion adducts, CID was carried out in the pre-mobility SRIG; for the charge-reduced ET products, CID was conducted in the postmobility SRIG (since the pre-mobility SRIG was used to perform ET).

The CCS distributions of doubly charged and chargereduced calcium and barium adducts of the two sets of isomers are shown in Figure 2. Generally, the doubly charged group II cation adducts for a given pair of isomers had very similar CCS distributions, while some of the ET products exhibited noticeable differences. For example, as illustrated in Figure 2a, the CCS distribution of $[\mathrm{FP} 1+\mathrm{Ca}]^{2+}$ was highly overlapped with that of $[\mathrm{FP} 5+\mathrm{Ca}]^{2+}$. Likewise, the $[\mathrm{FP} 1+\mathrm{Ba}]^{2+}$ and $[\mathrm{FP} 5+\mathrm{Ba}]^{2+}$ occupied similar ranges of CCS values. This observation was not entirely unexpected, as the group I cation adducts of FP isomers have previously been noted to have very similar CCSs. ${ }^{15,16}$ By contrast, the CCS distributions of the corresponding ET products were found to have substantially less overlap, with the $[\mathrm{FP} 5+\mathrm{Ca}]^{+\bullet}$ ion being noticeably larger than the $[\mathrm{FPI}+\mathrm{Ca}]^{\bullet}$ ion (Figure $2 \mathrm{~b}$ ). In comparison, the CCS distributions of $[\mathrm{FP} 1+\mathrm{Ba}]^{+\bullet}$ and $[\mathrm{FP} 5+\mathrm{Ba}]^{+\bullet}$ were not significantly different, although the $[\mathrm{FP} 5+\mathrm{Ba}]^{+\bullet}$ ion occupied a somewhat broader range of conformations as evidenced by the width of the CCS distribution. Similar general observations were noted for the FH1 and FH2. While FH1 and FH2 were not readily distinguishable as either their calcium or barium adducts (Figure $2 \mathrm{c}$ ), these oligosaccharide isomers were found to have remarkably distinct CCS distributions as the ET products of their calcium ion adducts (Figure 2d). Interestingly, the CCS distribution of $[\mathrm{FH} 2+\mathrm{Ca}]^{+\bullet}$ exhibited two features: a minor feature overlapped with the CCS distribution of $[\mathrm{FH} 1+\mathrm{Ca}]^{\bullet}$ and a major feature well-distinguished from the minor feature. In a similar fashion as the FP isomers, the charge reduced barium adducts of $\mathrm{FH}$ isomers did not exhibit distinct CCS distributions (Figure 2d). These observations serve to highlight the potential of ET reactions to result in isomerdistinguishing shifts in the CCSs of carbohydrate/metal ion
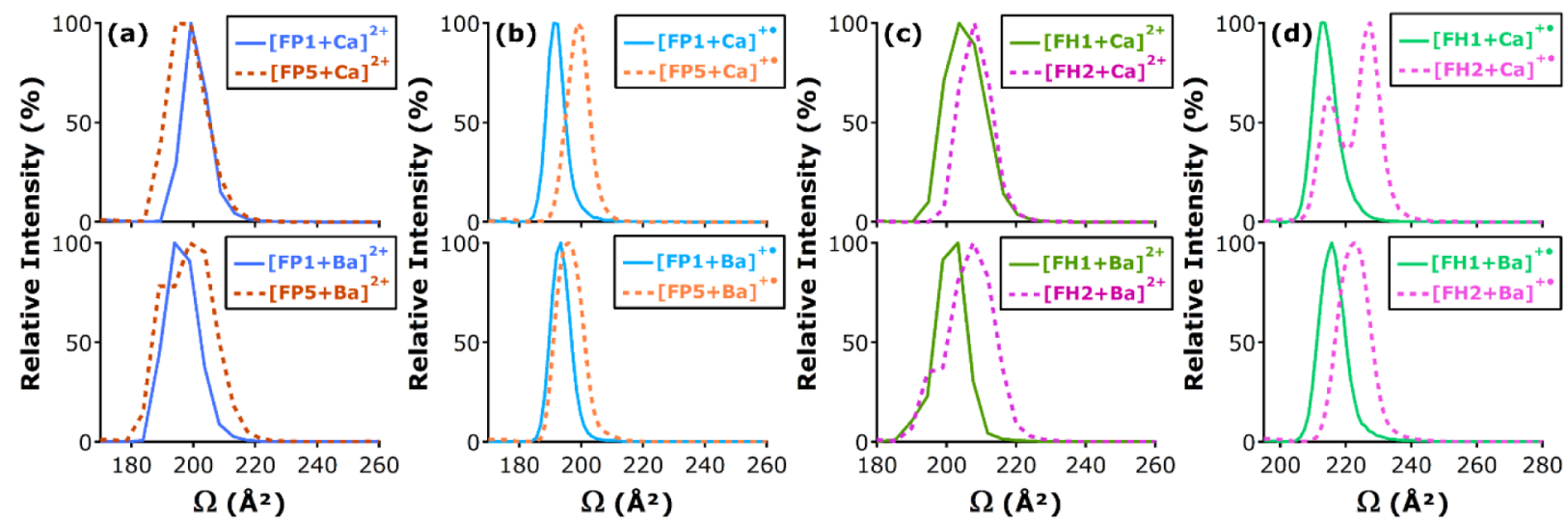

Figure 2. (a) Representative distributions of ion-neutral CCS values $(\Omega)$ for FP1 and FP5 as their calcium and barium ion adducts; (b) FP1 and FP5 as the ET products of their calcium and barium ion adducts; (c) FH1 and FH2 as their calcium and barium ion adducts; and (d) FH1 and FH2 as the ET products of their calcium and barium ion adducts. 
adducts with initially comparable sizes. Some of these examples are especially remarkable when considering that the shift occurs due to the transfer of a single electron to a given adduct. Future improvements in IMS resolution should render these observations increasingly viable for glycoanalysis.

The relevant ion-neutral CCSs and their uncertainties are more quantitatively compared in Figure 3. For FP1 and FP5, the largest CCS difference was noted between the chargereduced calcium ion adducts, with CCS values of $190.0 \pm 0.6$ $\AA^{2}$ for $[\mathrm{FP} 1+\mathrm{Ca}]^{+\bullet}$ and $198.3 \pm 0.2 \AA^{2}$ for $[\mathrm{FP} 5+\mathrm{Ca}]^{+\bullet}$ (Figure 3a). Interestingly, ET to the calcium ion adduct of FP1 resulted in a significant decrease in CCS (approximately $8.2 \AA^{2}$ ) when compared to the initial carbohydrate/metal ion adduct, while ET to the FP5 calcium ion adduct brought about a slight increase in CCS (approximately $2.3 \AA^{2}$ ) relative to the initial complex. Previous studies suggest that the bound cation is the principal site of electron acceptance in carbohydrate/metal ion adducts. ${ }^{24}$ In this case, any decrease in the size of the complex accompanying the addition of an electron to the valence shell of the bound metal may seem counterintuitive. That is, one might predict an increase in the size of the complex due to an increase in the effective ionic radius of $\mathrm{M}^{+} \bullet$ relative to $\mathrm{M}^{2+}$. Nevertheless, there is precedent for maintenance or reduction of carbohydrate/metal ion adduct CCS despite binding of successively larger group I cations. ${ }^{15,16}$ In these cases, increased metal ion radius may facilitate greater interaction with the carbohydrate and thus more compact structures. By contrast to the calcium ion adducts and ET products, the difference between the CCSs of FP1 and FP5 was approximately the same as both the barium ion adducts and their ET products. In both
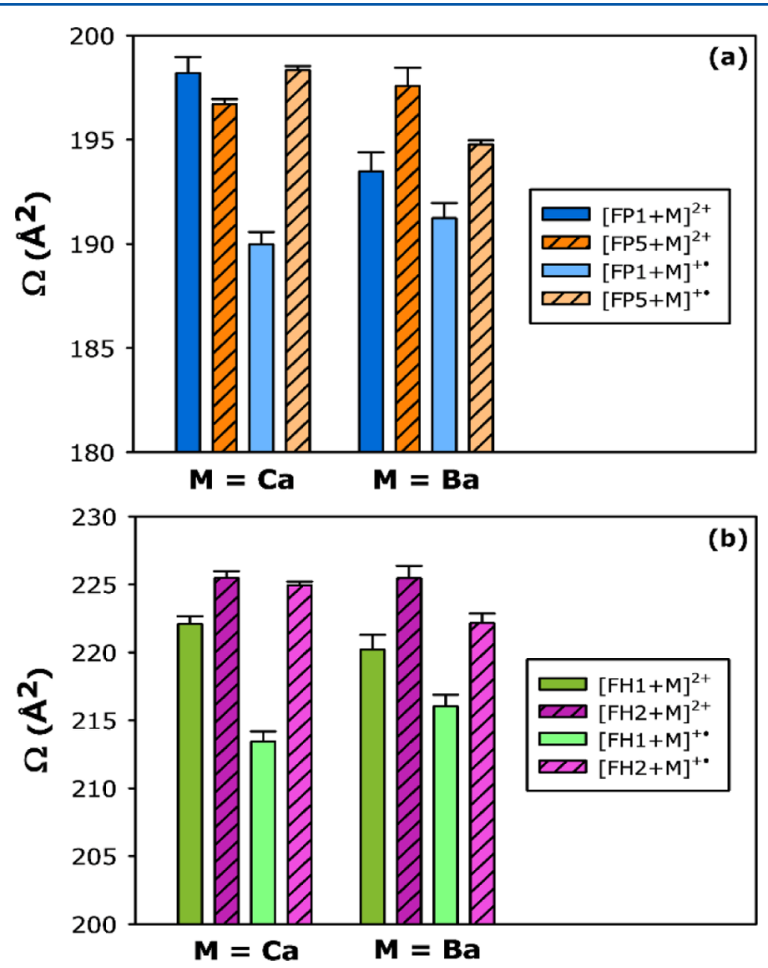

Figure 3. Average ion-neutral CCSs $(\Omega)$ for (a) FP1 and FP5 as calcium ion adducts, barium ion adducts, and ET products thereof and (b) FH1 and FH2 as calcium ion adducts, barium ion adducts, and ET products thereof. Error bars represent the standard error of the mean for four replicate measurements. The CCS of $[\mathrm{FH} 2+\mathrm{Ca}]^{+\bullet}$ was calculated for the more abundant of the two features seen in Figure $2 \mathrm{~d}$. cases, FP5 was the larger of the two isomers. The contrast between the calcium ion adducts and barium adducts with respect to their distinct size responses to ET illustrates the influence of the bound metal ion on isomer distinction of ET products, even where the initial adducts do not exhibit isomerresolving CCSs. This may be a reflection of the relatively greater change in effective ionic radius expected upon deposition of an electron into a vacant $4 \mathrm{~s}$ orbital of $\mathrm{Ca}^{2+}$ as opposed to a vacant $6 \mathrm{~s}$ orbital of $\mathrm{Ba}^{2+}$ (as the difference between energy levels is larger at lower principal quantum numbers).

As with the pentasaccharide isomers FP1 and FP5, the charged reduced calcium ion adducts also provided the largest size difference between the hexasaccharide isomers $\mathrm{FH} 1$ and $\mathrm{FH} 2$, with CCS values of $213.4 \pm 0.7 \AA^{2}$ for $[\mathrm{FH} 1+\mathrm{Ca}]^{+\bullet}$ and $224.9 \pm 0.3 \AA^{2}$ for $[\mathrm{FH} 2+\mathrm{Ca}]^{+\bullet}$ (Figure $3 \mathrm{~b}$ ). Notably, the transfer of a single electron to the $\mathrm{FH} 1$ calcium ion adduct resulted in an approximately $8.7 \AA^{2}$ reduction in the CCS of the complex (from $222.1 \pm 0.6 \AA^{2}$ for $[\mathrm{FH} 1+\mathrm{Ca}]^{2+}$ to $213.4 \pm 0.7$ $\AA^{2}$ for $\left.[\mathrm{FH} 1+\mathrm{Ca}]^{+\bullet}\right)$. By contrast, the CCS of the $\mathrm{FH} 2$ calcium adduct remained essentially unchanged by ET (from $225.5 \pm$ $0.5 \AA^{2}$ for $[\mathrm{FH} 2+\mathrm{Ca}]^{2+}$ to $\left.224.9 \pm 0.3 \AA^{2}[\mathrm{FH} 2+\mathrm{Ca}]^{+\bullet}\right)$. The CCSs of both FH1 and $\mathrm{FH} 2$ decreased as a result of ET to the barium ion adducts, and, as observed for the FP1 and FP5 isomers, the $\mathrm{FH} 1$ and $\mathrm{FH} 2$ isomers were similarly distinguishable as their barium ion adducts and corresponding ET products. Again, these results emphasize that carbohydrate metal ion adducts exhibit CCSs and shifts in CCS as a result of ET that are both isomer-dependent and cation-specific. Moreover, examples of isomer distinguishing conformational change resulting from the acceptance of a single electron indicate a role for both metal ion binding and gas-phase ion chemistry in the distinction of isomeric oligosaccharides by IMS. These are hitherto unprecedented observations.

In addition to the IMS studies, each FP and FH group II cation adduct and corresponding ET product was subjected to MS/MS using CID. These vibrational activation/dissociation spectra were acquired at selected collision energies, expressed here as potential differences $(\Delta U)$ through which the precursor ions were accelerated prior to collision. The CID spectra of $[\mathrm{FP} 1+\mathrm{Ba}]^{2+}$ and $[\mathrm{FP} 5+\mathrm{Ba}]^{2+}$ were very similar at each $\Delta U$ studied here. As shown in Figure 4a, at $\Delta U=20 \mathrm{~V}$, the CID spectra of $[\mathrm{FP} 1+\mathrm{Ba}]^{2+}$ and $[\mathrm{FP} 5+\mathrm{Ba}]^{2+}$ share essentially identical major fragment ion peaks, albeit in somewhat different relative intensities. This is highly characteristic of the vibrational activation/dissociation spectra of carbohydrate isomers. Because isomers only exhibit minor differences in the proportions of product ions, MS/MS methods often fail to unambiguously assign a structure. However, a strikingly different outcome was observed for the corresponding ET products of the FP1 and FP5 isomers as their barium-bound ET products. Figure $4 \mathrm{~b}$ shows the CID spectra of $[\mathrm{FP} 1+\mathrm{Ba}]^{+\bullet}$ and $[\mathrm{FP} 5+\mathrm{Ba}]^{+\bullet}$ at $\Delta U=$ $40 \mathrm{~V}$. Here, the most abundant fragment ion yielded by $[\mathrm{FP} 5+\mathrm{Ba}]^{+\bullet}$ was observed at $m / z 562.2$, which results from the loss of a fucose residue, a hexose residue, and $\mathrm{C}_{4} \mathrm{H}_{8} \mathrm{O}_{4}$ (likely via cross-ring cleavage). Only a small contribution of this fragment ion was observed in the $[\mathrm{FP} 1+\mathrm{Ba}]^{+\bullet}$ spectrum (relative intensity $<20 \%$ ). Conversely, the most abundant dissociation product of $[\mathrm{FP} 1+\mathrm{Ba}]^{+\bullet}$ was observed at $\mathrm{m} / z$ 359.0, which includes the losses described for $m / z 562.1$, with further loss of an $\mathrm{N}$-acetylglucosamine residue. For $[\mathrm{FP} 5+\mathrm{Ba}]^{+\bullet}$, the relative intensity of $m / z 359.0$ was $<15 \%$. Major peaks in the $[\mathrm{FP} 1+\mathrm{Ba}]^{+\bullet}$ spectrum (e.g., $m / z 502.1,544.1,648.1,708.1$, and 

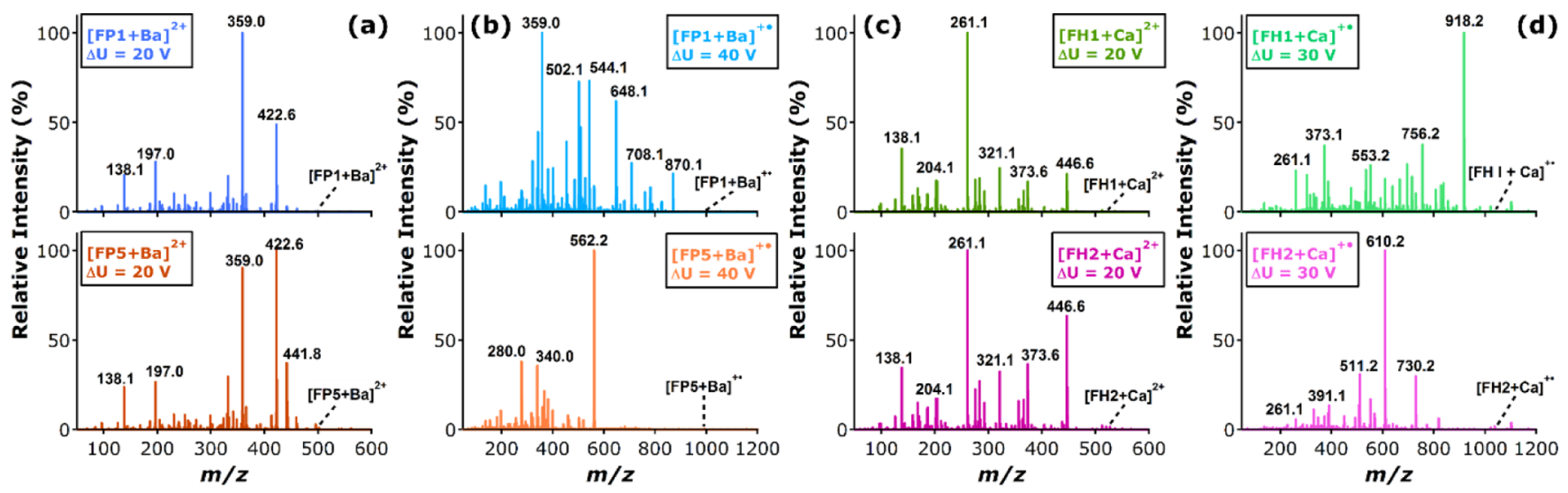

Figure 4. MS/MS spectra produced by (a) low-energy transmission type CID of FP1 and FP5 as barium ion adducts and (b) the ET products thereof and (c) FH1 and $\mathrm{FH} 2$ as calcium ion adducts and the (d) ET products thereof. In each case, the potential difference $(\Delta U)$ used to accelerate precursor ions has been indicated.

870.1 ) were either absent or showed $<10 \%$ relative intensity for $[\mathrm{FP} 5+\mathrm{Ba}]^{+\bullet}$. These fragments can serve as diagnostic ion signals in distinguishing the two isomers.

MS/MS studies were performed on the $\mathrm{FH} 1$ and $\mathrm{FH} 2$ isomers, as well. At $\Delta U=20 \mathrm{~V}$, CID of $[\mathrm{FH} 1+\mathrm{Ca}]^{2+}$ and $[\mathrm{FH} 2+\mathrm{Ca}]^{2+}$ resulted in many of the same product ions (Figure 4c). Again, the extent of discrimination afforded by direct CID of the metal ion adducts was limited to changes in the relative intensities of dissociation products with the same $\mathrm{m} / \mathrm{z}$. However, in accord with the results described above, ET products of the calcium adducted $\mathrm{FH} 1$ and $\mathrm{FH} 2$ isomers yielded fragmentation spectra with high potential for isomer distinction (Figure $4 \mathrm{~d}$ ). For $\Delta U=30 \mathrm{~V}$, the most abundant peak in the spectra of $[\mathrm{FH} 1+\mathrm{Ca}]^{+\bullet}$ was $\mathrm{m} / z$ 918.2, which results from a loss of $\mathrm{C}_{4} \mathrm{H}_{8} \mathrm{O}_{4}$ from the charge reduced precursor. However, the most abundant ion in the spectrum of $[\mathrm{FH} 1+\mathrm{Ca}]^{+\bullet}$ was $m / z 610.2$, a product of the losses of a fucose residue, a hexose residue, and $\mathrm{C}_{4} \mathrm{H}_{8} \mathrm{O}_{4}$ from the charge-reduced precursor. A fragment ion of $m / z 918.2$ was not observed in the corresponding spectrum for the $\mathrm{FH} 2$ isomer, and $<15 \%$ relative abundance of $\mathrm{m} / \mathrm{z} 610.2$ was observed in the FH1 spectrum. On the whole, these initial MS/MS studies demonstrate significant potential for the discrimination of oligosaccharide isomers that differ only in the attachment point of a single monosaccharide. Such dissimilar CID spectra for carbohydrate isomers have not previously been observed.

In conclusion, this work demonstrates the first exploitation of carbohydrate/metal ion interactions in concert with gas-phase ET reactions to enhance the capacity of both IMS and MS/MS to discriminate native isomeric oligosaccharides. This is attributed to the acceptance of an electron by the bound metal ion, which in turn brings about analytically useful transformations in the ion-neutral CCSs and vibrational activation/dissociation chemistries of the adducts.

\section{AUTHOR INFORMATION}

\section{Corresponding Author}

*E-mail: edodds2@unl.edu. Tel: 1-402-472-3592.

\section{Notes}

The authors declare no competing financial interest.

\section{ACKNOWLEDGMENTS}

This work was supported by funding from the University of Nebraska, which was provided in part by the Nebraska Tobacco
Settlement Biomedical Research Development Fund. The authors thank Abby S. Gelb, Forouzan Aboufazeli, and Abbegayle J. Dodds for input on the manuscript.

\section{REFERENCES}

(1) Vanderschaeghe, D.; Festjens, N.; Delanghe, J.; Callewaert, N. Biol. Chem. 2010, 391, 149-161.

(2) Rakus, J. F.; Mahal, L. K. Annu. Rev. Anal. Chem. 2011, 4, 367392

(3) Zaia, J. Mass Spectrom. Rev. 2004, 23, 161-227.

(4) Kailemia, M. J.; Ruhaak, L. R.; Lebrilla, C. B.; Amster, I. J. Anal. Chem. 2013, 86, 196-212.

(5) Pabst, M.; Altmann, F. Proteomics 2011, 11, 631-643.

(6) Kolli, V.; Schumacher, K. N.; Dodds, E. D. Bioanalysis 2015, 7, 113-131.

(7) Lapthorn, C.; Pullen, F.; Chowdhry, B. Z. Mass Spectrom. Rev. 2013, 32, 43-71.

(8) Huang, Y.; Gelb, A. S.; Dodds, E. D. Curr. Metabolomics 2013, 1, 291-305.

(9) Williams, J. P.; Grabenauer, M.; Holland, R. J.; Carpenter, C. J.; Wormald, M. R.; Giles, K.; Harvey, D. J.; Bateman, R. H.; Scrivens, J. H.; Bowers, M. T. Int. J. Mass Spectrom. 2010, 298, 119-127.

(10) Zhu, F.; Lee, S.; Valentine, S. J.; Reilly, J. P.; Clemmer, D. E. J. Am. Soc. Mass Spectrom. 2012, 23, 2158-2166.

(11) An, H. J.; Lebrilla, C. B. Mass Spectrom. Rev. 2011, 30, 560-578.

(12) Zhou, W.; Hakansson, K. Curr. Proteomics 2011, 8, 297-308.

(13) Cancilla, M. T.; Penn, S. G.; Carroll, J. A.; Lebrilla, C. B. J. Am. Chem. Soc. 1996, 118, 6736-6745.

(14) Zhu, F.; Glover, M. S.; Shi, H.; Trinidad, J. C.; Clemmer, D. E. J. Am. Soc. Mass Spectrom. 2015, 26, 25-35.

(15) Fenn, L. S.; McLean, J. A. Phys. Chem. Chem. Phys. 2011, 13, 2196-2205.

(16) Huang, Y.; Dodds, E. D. Anal. Chem. 2013, 85, 9728-9735.

(17) Guerrero, A.; Lebrilla, C. B. Int. J. Mass Spectrom. 2013, 354, $19-25$.

(18) Han, L.; Costello, C. E. J. Am. Soc. Mass Spectrom. 2011, 22, 997-1013.

(19) Varki, A.; Cummings, R. D.; Esko, J. D.; Freeze, H. H.; Stanley, P.; Marth, J. D.; Bertozzi, C. R.; Hart, G. W.; Etzler, M. E. Proteomics 2009, 9, 5398-5399.

(20) Harvey, D. J.; Merry, A. H.; Royle, L.; Campbell, M. P.; Dwek, R. A.; Rudd, P. M. Proteomics 2009, 9, 3796-3801.

(21) Syka, J. E. P.; Coon, J. J.; Schroeder, M. J.; Shabanowitz, J.; Hunt, D. F. Proc. Natl. Acad. Sci. U.S.A. 2004, 101, 9528-9533.

(22) Bush, M. F.; Campuzano, I. D. G.; Robinson, C. V. Anal. Chem. 2012, 84, 7124-7130.

(23) Gelb, A. S.; Jarratt, R. E.; Huang, Y.; Dodds, E. D. Anal. Chem. 2014, 86, 11396-11402. 
(24) Huang, Y.; Pu, Y.; Yu, X.; Costello, C. E.; Lin, C. J. Am. Soc. Mass Spectrom. 2014, 25, 1451-1460. 